\title{
Evaluation of analgesic regimens in total knee arthroplasty, retrospective study
}

\author{
Serkan Tulgar, ${ }^{1}$ Onur Selvi, ${ }^{1}$ Ozgur Senturk, ${ }^{1}$ Talat Ercan Serifsoy, ${ }^{1}$ \\ Selim Sanel, ${ }^{2}$ Sertac Meydaneri'² \\ ${ }^{1}$ Department of Anesthesiology and Reanimation, Maltepe University Faculty of Medicine, Istanbul, Turkey \\ ${ }^{2}$ Department of Ortopedy and Travmatology, Maltepe University Faculty of Medicine, Istanbul, Turkey
}

\begin{abstract}
OBJECTIVE: Analgesic therapies have an immense role in early rehabilitation period after total knee arthroplasty (TKA) and multimodal approaches should be considered as the first choice of treatment. In this retrospective study, the aim was to evaluate the effectiveness of multimodal analgesic therapies for TKA, including femoral nerve block (FNB) and patient controlled analgesia (PCA).

METHODS: The data of 79 patients who underwent TKA between January and December 2016 were retrospectively evaluated. In all, 63 patients met the inclusion criteria. Hemodynamic records and Visual Analogue Scale (VAS) pain scores for postoperative 0, 2, 4, 6, 9, and 12 hours were evaluated and patients were separated into 3 groups. Group 1: FNB with $0.25 \%$ bupivacaine, Group 2: FNB with $0.166 \%$ bupivacaine, and Group 3: No FNB.

RESULTS: The average age of the patients was $64.3 \pm 14.9$ years and average body mass index (BMI) was $32.5 \pm 5.3 \mathrm{~kg} / \mathrm{m}^{2}$. There was no statistical difference between groups in age, gender, American Society of Anesthesiologists (ASA) classification of physical health scores, BMI, or anesthesia type $(p<0.05)$. When VAS scores at postoperative time intervals were compared, there was a statistically significant difference between Group 1 and Group 2 ( $p>0.05$ ). When difference between Groups 1 and 3 and Groups 2 and 3 were compared, the difference was statistically significant for VAS 0 ( $p>0.05)$. Additional analgesic use was highest in Group 3.
\end{abstract}

CONCLUSION: This study demonstrated that FNB significantly decreases postoperative pain intensity and additional analgesia requirement in patients undergoing TKA. A concentration of $0.166 \%$ bupivacaine is as effective as a concentration of $0.25 \%$ when used as part of a multimodal analgesia regimen in TKA.

Keywords: Analgesia; femoral block; knee arthroplasty.

$\mathrm{P}$ ostoperative pain is a common complaint after total knee arthroplasty (TKA) [1]. Appropriate management of this postoperative pain is im- portant during the early rehabilitation period [2]. Postoperative analgesia after TKA can be administered using several techniques, including intrave-

Received: January 26, 2017 Accepted: June 12, 2017 Online: August 25, 2017

Correspondence: Dr. Serkan TULGAR. Maltepe Universitesi Tip Fakultesi Hastanesi, Feyzullah Caddesi, No: 39, Maltepe, Istanbul, Turkey.

Tel: +90 216 - 3999750 e-mail: serkantulgar.md@gmail.com

(c) Copyright 2017 by Istanbul Northern Anatolian Association of Public Hospitals - Available online at www.kuzeyklinikleri.com 
nous (IV) analgesics, neuraxial blocks, and peripheral nerve blocks [2-5].

The use of a femoral nerve block (FNB) in patients undergoing TKA has increased recently, as it allows for early rehabilitation, and decreases postoperative pain, the use of opioids, and the length of hospital stay [6-8]. Some authors have stated that FNB is the gold standard for analgesia in patients undergoing TKA $[6,9,10]$.

If physically possible, it is advised to perform all peripheral nerve blocks under ultrasonographic guidance in order to increase safety and the success of the block [11-13]. However, when ultrasonography is not available, performance of peripheral nerve block using a traditional nerve stimulator (NS) is also a safe technique [14].

Studies evaluating the use of FNB in patients undergoing TKA in our country are scarce [15]. While patients traditionally received IV patientcontrolled analgesia (PCA) after TKA at our institute, FNB has been used as a component of multimodal analgesia for the last year.

In this retrospective study, different modalities of postoperative analgesia administered to patients undergoing TKA at our institute were evaluated.

\section{MATERIALS AND METHODS}

After receiving institutional review board approval, the medical data of all patients who underwent TKA between January 1, 2016 and December 31, 2016 were retrospectively reviewed. Patient consent was not sought, as data were collected without patient identifiers.

Patient files were reviewed, and patients with the following characteristics were excluded: patients who underwent neuraxial anesthesia, bilateral TKA, American Society of Anesthesiologists (ASA) classification of physical health status IV, emergency case, psychiatric or neurological pathology that would interfere with evaluation of pain, and those with missing data. PCA follow-up forms were used for documentation of pain; therefore, patients with missing PCA forms were also excluded. Patient age, gender, body mass index (BMI) plus peroperative and postoperative analgesia regimens were noted.

All patients' peroperative analgesia regimens were analyzed. Patients with analgesia regimen that differed from standard protocol as detailed below were also excluded. The routine perioperative analgesia protocol of the institute is as follows: $0.1 \mathrm{mg} /$ $\mathrm{kg}$ morphine, $1 \mathrm{~g}$ IV paracetamol, and $20 \mathrm{mg}$ tenoxicam administered at least 1 hour before end of surgery. PCA is commenced in postanesthetic care unit (PACU). PCA included: $400 \mathrm{mg}$ tramadol in 100 $\mathrm{mL} 0.09$ sodium chloride solution to create $4 \mathrm{mg} /$ $\mathrm{mL}$ concentration of tramadol. PCA was set to deliver $10 \mathrm{mg}$ infusion per hour plus $10 \mathrm{mg}$ bolus with 20-minute lock time. PCA forms were completed in PACU and follow-up in wards included hourly recording of VAS score and hemodynamic parameters. Postoperatively, IV paracetamol $3 \times 1 \mathrm{~g}$ was ordered for all patients by the surgical team. All other drugs used for analgesia were noted on the PCA forms. These drugs were cross-checked with electronic orders. Intramuscular diclofenac was used as rescue analgesia in all patients with a VAS score over 4 and $50 \mathrm{mg}$ IV meperidine was administered if VAS score remained above 4 after 2 hours.

VAS scores were noted at $0,2,4,6,9$, and 12 hours after the patient was transferred from PACU to ward. The maximum VAS score was noted for the respective time frame. For standardization, VAS evaluation until the 12th hour was used, as PCA was terminated at different times after that point. When the data were retrospectively reviewed, the patients were separated into 3 groups according to analgesia protocol:

Group 1 ( $0.25 \%$ bupivacaine): Twenty $\mathrm{mL}$ of mixture containing $10 \mathrm{~mL}$ bupivacaine (Marcaine 0.5\%; Hospira, Inc./Pfizer, Inc., New York, NY, USA) + $5 \mathrm{~mL}$ lidocaine (Aritmal 2\%; Osel İlaç Sanayi ve Tic. A.Ş., Istanbul, Turkey) $+5 \mathrm{~mL}$ physiological saline.

Group 2 (0.166\% bupivacaine): A solution of $24 \mathrm{~mL}$ was prepared ( $8 \mathrm{~mL}$ bupivacaine [Marcaine $0.5 \%$ ] $+5 \mathrm{~mL}$ lidocaine [Aritmal 2\%] $+11 \mathrm{~mL}$ physiological saline) and of this mixture, $20 \mathrm{~mL}$ was injected for FNB.

Group 3: No femoral block. 


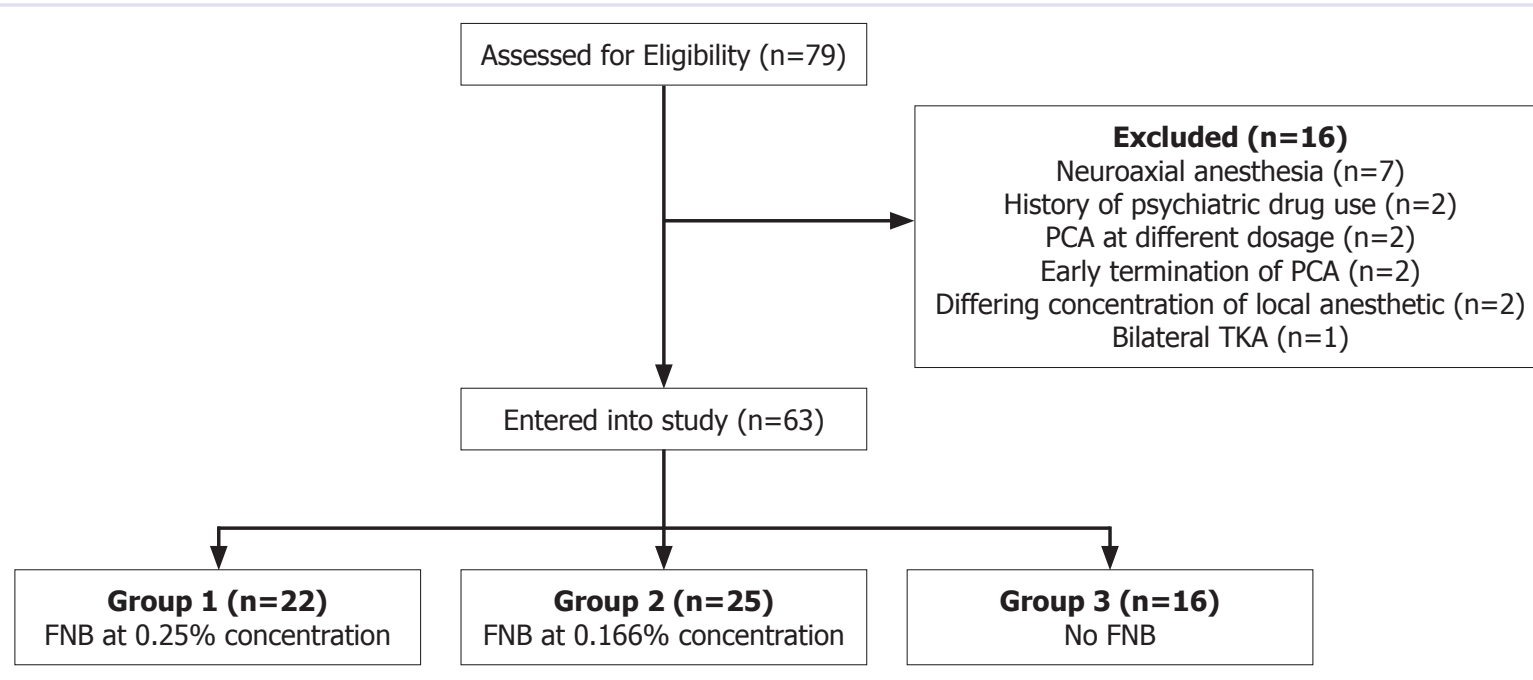

FIGURE 1. Flowchart of the study methodology. FNB: Femoral nerve block; PCA: Patient-controlled analgesia.

The local anesthetic concentration and mixture preparation used was according to the preference of the anesthesiologist.

In all cases where FNB was used, it was administered at the end of surgery under conscious sedation. A nerve stimulator (STIMPOD NMS 400; Xavant Technology, Silverton, Pretoria, South Africa) and an insulated Stimuplex (B. Braun Medical, Inc, Bethlehem, PA, USA) needle were used. Antiseptic solution was used to prepare the skin of the femoral area of the ipsilateral side of the TKA. An insulated 5-cm needle was inserted immediately lateral to the femoral artery at the femoral skin crease. After negative aspiration to check for blood, anesthetic agents were injected into the location where $0.25-0.5 \mathrm{~mA}$ twitch of the quadriceps muscle (lateral and medial branches) was observed.

\section{Statistical analysis}

SPSS for Windows, Version 16.0 (SPSS, Inc, Chicago, IL, USA) software was used for statistical analysis. Mean $\pm \mathrm{SD}$ was used for descriptive statistics. Ratios were compared using chi-square test. Qualitative data was compared using Fisher's exact test. Continuous variables were compared using one-way analysis of variance with post hoc Tukey analysis. A p value of $<0.05$ was regarded as statistically significant.

\section{RESULTS}

The medical files of 79 patients were retrospectively reviewed. Seven patients who underwent spinal or spinal-epidural anesthesia, 2 patients with a history of psychiatric drug use, 2 patients who used different dose of PCA, 2 patients with different concentration of local anesthetic agents, 2 patients with early termination of PCA due to technical issues, and 1 patient who underwent bilateral TKA were excluded from the study. The data of 63 patients (49 female, 14 male) were included in the study. Inclusion flowchart is provided in Figure 1.

The average age of the study patients was $64.3 \pm 14.9$ years and average BMI was $32.5 \pm 5.3$ $\mathrm{kg} / \mathrm{m}^{2}$. The age, gender, ASA score, BMI, anesthesia type, and surgical time for all groups can be seen in Table 1. Comparison of these data between groups revealed no statistically significant difference $(\mathrm{p}>0.05)$.

When VAS scores at 0, 2, 4, 6, 9, and 12 hours were compared there was a statistically significant difference between groups. Post hoc analysis revealed no difference between Groups 1 and 2 at any time period. However, when difference between Groups 1 and 3 and Groups 2 and 3 were compared, the difference was statistically significantly for VAS $0(p>0.05)$ and extremely significant for other VAS time frames $(p<0.01)$. 
TABLE 1. Patient demographics

\begin{tabular}{lccccc} 
& All patients $(\mathrm{n}=63)$ & Group 1 $(\mathrm{n}=22)$ & Group 2 $(\mathrm{n}=26)$ & Group 3 $(\mathrm{n}=16)$ & $\mathrm{p}$ \\
\hline Age (years) & $64.37 \pm 14.91$ & $68.59 \pm 7.66$ & $63.60 \pm 15.90$ & $59.75 \pm 19.56$ & $0.188^{*}$ \\
Male/female $(\mathrm{n})$ & $49 / 14$ & $20 / 2$ & $18 / 7$ & $11 / 5$ & $0.177^{* *}$ \\
ASA I/II/III $(\mathrm{n})$ & $10 / 25 / 28$ & $0 / 10 / 12$ & $5 / 10 / 10$ & $5 / 5 / 6$ & $0.079^{* *}$ \\
Average BMI kg/m & $32.50 \pm 5.37$ & $33.89 \pm 5.73$ & $31.64 \pm 4.89$ & $31.95 \pm 5.55$ & $0.328^{*}$ \\
Surgical time (minutes) & $126.8 \pm 15.9$ & $127 \pm 14.5$ & $122.6 \pm 16.3$ & $125.8 \pm 17.2$ & $0.616^{*}$ \\
\hline
\end{tabular}

ASA: American Society of Anesthesiologists classification of physical health; BMI: Body mass index. *Analysis of variance; ** Fisher's exact test probability.

TABLE2. Average postoperative Visual Analogue Scale pain score according to group

\begin{tabular}{lcccc} 
& Group 1 & Group 2 & Group 3 & $p$ \\
\hline 0 hour & $2.77 \pm 1.15$ & $2.84 \pm 0.94$ & $4.44 \pm 1.96$ & $<0.001$ \\
$2^{\text {nd }}$ hour & $2.23 \pm 1.15$ & $1.80 \pm 0.65$ & $4.25 \pm 1.69$ & $<0.001$ \\
$4^{\text {th }}$ hour & $1.68 \pm 1.08$ & $1.60 \pm 0.65$ & $3.62 \pm 1.10$ & $<0.001$ \\
$6^{\text {th }}$ hour & $1.41 \pm 1.08$ & $1.48 \pm 0.71$ & $4.0 \pm 1.41$ & $<0.001$ \\
$9^{\text {th }}$ hour & $1.64 \pm 1.04$ & $1.40 \pm 0.81$ & $4.25 \pm 1.39$ & $<0.001$ \\
$12^{\text {th }}$ hour & $1.45 \pm 0.85$ & $1.20 \pm 0.81$ & $4.0 \pm 1.75$ & $<0.001$
\end{tabular}

VAS score comparison between groups is shown in Table 2. VAS scores and SD are illustrated in Figure 2. Table 3 reports the postoperative analgesia protocol used by the orthopedics clinic in patients who required additional analgesia. There was an extremely significant statistical difference between groups when the use of additional anesthesia was analyzed. When compared to other groups, Group 3 had 104.2\% more patients receiving additional analgesic, which was statistically significant. There was no difference between Groups 1 and 2 .
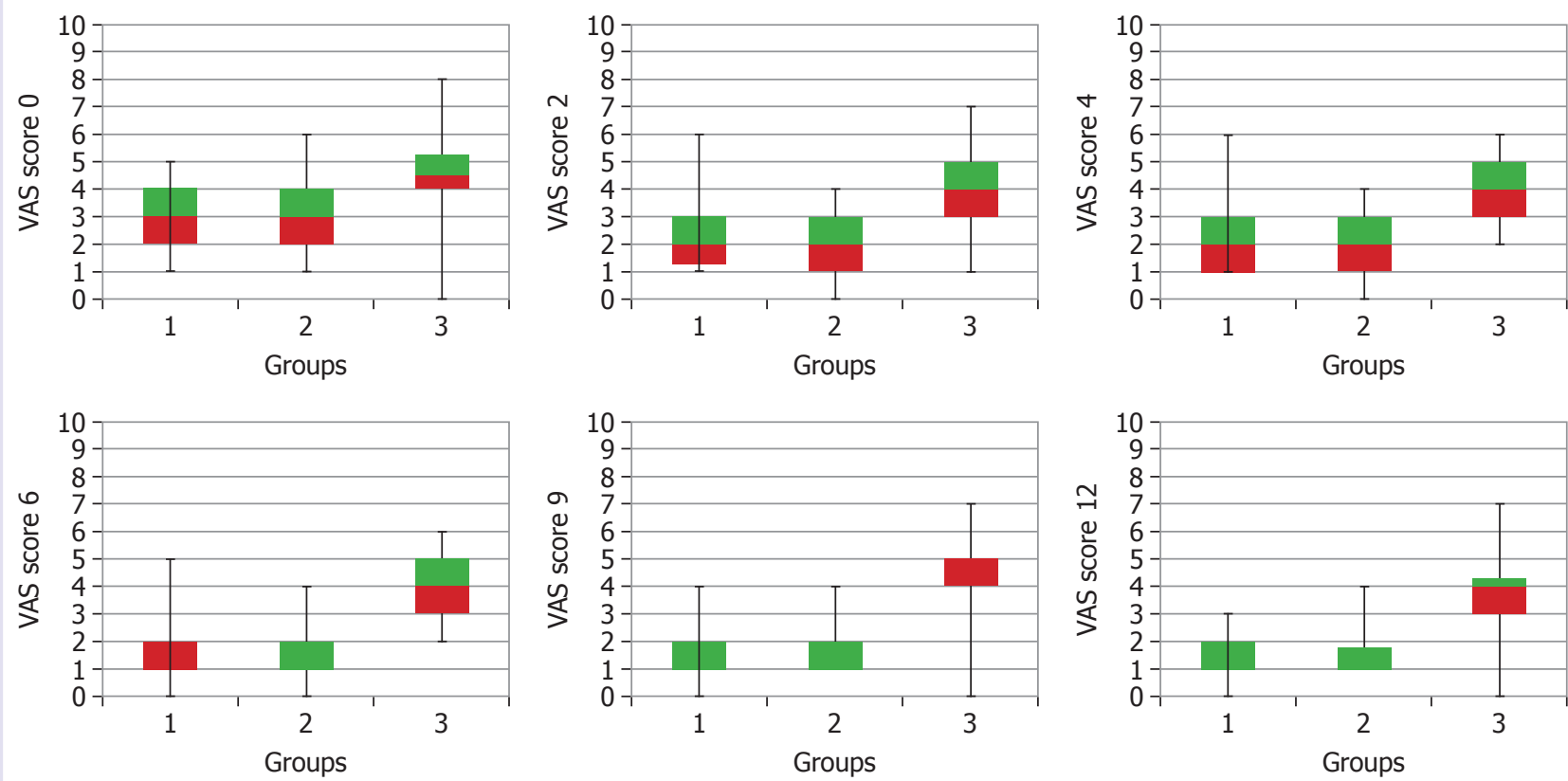

FIGURE2. Postoperative Visual Analogue Scale scores at postoperative time intervals according to group. Green represents the maximum $3^{\text {rd }}$ quartile (3/4) and red represents the minimum first quartile (1/4). VAS: Visual Analogue Scale of pain. 
TABLE 3. Additional analgesia requirements of the patients by group

\begin{tabular}{lcccc} 
& Group 1 (PD) & Group 2 (PD) & Group 3 (PD) & $p$ \\
\hline No additional analgesia & $16(+27.3 \%)$ & $18(+26 \%)$ & $2(-78.1 \%)$ & $<0.001$ \\
Additional analgesia required & $6(-36.4 \%)$ & $7(-34.7 \%)$ & $14(+104.2 \%)$ & \\
Diclofenac & 3 & 5 & 5 & \\
Diclofenac and meperidine & 3 & 2 & 9 & \\
\hline
\end{tabular}

PD: Percentage deviation.

\section{DISCUSSION}

In this study we retrospectively reviewed the data of patients who underwent TKA at our institute. Patients who had FNB administered were found to have less pain during the first postoperative 12 hours and required less analgesic agent. Bupivacaine at $0.25 \%$ and $0.166 \%$ concentrations was used. When the 2 groups with different concentrations were compared, there was no difference between patient VAS scores or additional analgesic requirement.

The administration of peripheral nerve block under ultrasound (US) guidance is the safest and most accepted method. The authors do not claim that peripheral nerve stimulator (PNS) is as successful and safe as US guidance. The aim of this study was not to compare PNS with US, but to evaluate the use of different concentrations of bupivacaine in FNB.

Postoperative analgesia in TKA patients has been the subject of much research. While epidural analgesia was once considered the criterion standard for TKA, this has now been replaced with FNB [9]. However, FNB may lead to some undesirable effects. The most important of these is FNBrelated quadriceps weakness $[2,16,17]$. It has been suggested that blocking the femoral nerve more distally within the adductor canal could help prevent this effect $[4,18,19]$. However, adductor canal block is an advanced nerve block technique that is performed using US guidance. In our institute, we prefer to use FNB, as it is easily performed using a PNS and US is not available. Although we did not routinely evaluate quadriceps weakness in our patients, no falls were reported in their files.
There are a limited number of studies regarding FNB use in TKA in our country. Sahin et al. [15] performed US-guided FNB using $40 \mathrm{~mL}$ of $0.5 \%$ bupivacaine after $\mathrm{T} 12$ regression of spinal anesthesia. Their study reported significant decrease in opioid consumption with sufficient and effective analgesia for up to 48 hours. In another study from our country, $20 \mathrm{~mL}$ of $0.25 \%$ bupivacaine was administered near the femoral nerve with US and/or PNS guidance followed by placement of a catheter. Continuous bupivacaine was administered at a concentration of $0.125 \%$ with sufficient postoperative analgesia observed [20]. They reported no difference in analgesia effectiveness, length, or procedure performance (time, success, etc.) between patients undergoing FNB with or without US guidance.

These 2 studies differ from ours in design and objective. However, the concentration and volume of bupivacaine should be discussed. Sahin et al. used $40 \mathrm{~mL}$ of $0.5 \%$ bupivacaine, a larger volume and concentration compared with our study. Aytac et al. [20] used $0.25 \%$ of bupivacaine concentration. We were unable to find a study that used our low concentration of $0.166 \%$ bupivacaine as the single shot or initiation dose. All patients in our study had a single injection for FNB. Our findings have demonstrated effective analgesia at low volume and concentration.

The success of FNB is dependent on numerous factors, including the concentration and volume of the local anesthetic. There are several studies comparing different concentrations of bupivacaine for FNB [21,22]. In a randomized, double-blind study to determine the minimum effective concentration 
of bupivacaine in US-guided FNB for knee meniscectomy, bupivacaine was administered at concentrations ranging from $0.15 \%$ to $0.35 \%$. Effective concentration (EC) 50 and EC90 were found to be $0.160 \%$ and $0.271 \%$, respectively [21]. The concentrations of bupivacaine used in our study were within this range.

Many studies have evaluated the effectiveness of FNB or have compared it with another analgesia method in TKA. A large majority of these studies have reported the use of $0.25 \%$ bupivacaine for single dose FNB $[3,8,15,23,24]$. When the catheter was placed near the femoral nerve, bolus of $0.25 \%$ bupivacaine was followed by $0.125 \%$ concentration as a continuous infusion [20, 25-27]. Most studies report $0.25 \%$ concentration of bupivacaine as the single shot or initiation dose. We have demonstrated that a single injection FNB with bupivacaine concentrations of $0.166 \%$ and $0.25 \%$ administered as part of a multimodal analgesia regimen are just as effective.

Our study has some limitations. The primary limitation is performing FNB without US guidance. Another is that the presence and duration of motor block, quadriceps weakness, and fall risk scores plus VAS scores after the 12th hour were unavailable from patient files. Determination of tramadol consumption in patients with tramadol PCA would have added value to the study. However, analysis of rescue analgesia use partially decreases the effect of this limitation.

Although there was no statistical difference in the demographics and surgical time between groups, the study's retrospective design means that the groups may not be similar with regard to surgical trauma and severity or degree of damage to the knee. A well-designed prospective study that takes all demographics and confounders into account is required for a better level of evidence.

\section{Conclusion}

This study has demonstrated that FNB used in addition to our routine clinical analgesia protocol significantly decreased postoperative pain intensity and additional analgesia requirement in patients who underwent TKA. A concentration of $0.166 \%$ bupivacaine was as effective as a concentration of $0.25 \%$ when used as part of multimodal analgesia regimen in TKA.

\section{Conflict of Interest: None declared.}

Financial Disclosure: The authors declared that this study has received no financial support.

Authorship contributions: Concept - S.T., O.S.; DesignS.T., O.S.; Supervision - S.T., T.E.S.; Materials - S.M., S.T., O.S., S.S.; Data collection \&/or processing - S.M., S.S., S.T.; Analysis and/or interpretation - S.T. ; Literature search - S.T.; Writing - S.T.; Critical review - S.T., O.S.

\section{REFERENCES}

1. Specht K, Kjaersgaard-Andersen P, Pedersen BD. Patient experience in fast-track hip and knee arthroplasty--a qualitative study. J Clin Nurs 2016;25:836-45.

2. Li D, Tan Z, Kang P, Shen B, Pei F. Effects of multi-site infiltration analgesia on pain management and early rehabilitation compared with femoral nerve or adductor canal block for patients undergoing total knee arthroplasty: a prospective randomized controlled trial. Int Orthop 2017;41:75-83.

3. Shin HJ, Soh JS, Lim HH, Joo B, Lee HW, Lim HJ. In-plane three-step needle insertion technique for ultrasound-guided continuous femoral nerve block after total knee arthroplasty: a retrospective review of 488 cases. Korean J Anesthesiol 2016;69:587-91.

4. Joe HB, Choo HS, Yoon JS, Oh SE, Cho JH, Park YU. Adductor canal block versus femoral nerve block combined with sciatic nerve block as an anesthetic technique for hindfoot and ankle surgery: A prospective, randomized noninferiority trial. Medicine (Baltimore) 2016;95:e5758.

5. Kodaka M, Minayoshi H, Ichikawa J, Nishiyama K, Komori M. A Prospective, Randomized, Blinded Trial to Compare Continuous Epidural Block and Femoral Nerve Block for Total Knee Arthroplasty. J Anesth Clin Res 2014;05:410-5.

6. Tanikawa H, Sato T, Nagafuchi M, Takeda K, Oshida J, Okuma $\mathrm{K}$. Comparison of local infiltration of analgesia and sciatic nerve block in addition to femoral nerve block for total knee arthroplasty. J Arthroplasty 2014;29:2462-7.

7. Sakai N, Inoue T, Kunugiza Y, Tomita T, Mashimo T. Continuous femoral versus epidural block for attainment of $120^{\circ}$ knee flexion after total knee arthroplasty: a randomized controlled trial. J Arthroplasty 2013;28:807-14.

8. Emerson RH Jr, Barrington JW, Olugbode O, Lovald S, Watson H, Ong K. Femoral Nerve Block Versus Long-Acting Wound Infiltration in Total Knee Arthroplasty. Orthopedics 2016;39:e449-55.

9. Hadzic A, Houle TT, Capdevila X, Ilfeld BM. Femoral nerve block for analgesia in patients having knee arthroplasty. Anesthe- 
siology 2010;113:1014-5.

10. Bauer MC, Pogatzki-Zahn EM, Zahn PK. Regional analgesia techniques for total knee replacement. Curr Opin Anaesthesiol 2014;27:501-6.

11. Ratnawat A, Bhati FS, Khatri C, Srinivasan B, Sangwan P, Chouhan DP. Comparative study between nerve stimulator guided technique and ultrasound guided technique of supraclavicular nerve block for upper limb surgery. Int J Res Med Sci 2016;4:2101-6.

12. Beaudoin FL, Nagdev A, Merchant RC, Becker BM. Ultrasound-guided femoral nerve blocks in elderly patients with hip fractures. Am J Emerg Med 2010;28:76-81.

13. Fredrickson MJ, Kilfoyle DH. Neurological complication analysis of 1000 ultrasound guided peripheral nerve blocks for elective orthopaedic surgery: a prospective study. Anaesthesia 2009;64:836-44.

14. Wang F, Liu LW, Hu Z, Peng Y, Zhang XQ, Li Q. Ultrasound and nerve stimulator guided continuous femoral nerve block analgesia after total knee arthroplasty: a multicenter randomized controlled study. Rev Bras Anestesiol 2015;65:14-20.

15. Sahin L, Korkmaz HF, Sahin M, Atalan G. Ultrasound-guided single-injection femoral nerve block provides effective analgesia after total knee arthroplasty up to 48 hours. Agri 2014;26:113-8.

16. Jaeger P, Nielsen ZJ, Henningsen MH, Hilsted KL, Mathiesen O, Dahl JB. Adductor canal block versus femoral nerve block and quadriceps strength: a randomized, double-blind, placebocontrolled, crossover study in healthy volunteers. Anesthesiology 2013;118:409-15.

17. Dong CC, Dong SL, He FC. Comparison of Adductor Canal Block and Femoral Nerve Block for Postoperative Pain in Total Knee Arthroplasty: A Systematic Review and Meta-analysis. Medicine (Baltimore) 2016;95:e2983.

18. Shah NA, Jain NP. Is continuous adductor canal block better than continuous femoral nerve block after total knee arthroplasty? Effect on ambulation ability, early functional recovery and pain control: a randomized controlled trial. J Arthroplasty 2014;29:2224-9.
19. Memtsoudis SG, Yoo D, Stundner O, Danninger T, Ma Y, Poultsides L, et al. Subsartorial adductor canal vs femoral nerve block for analgesia after total knee replacement. Int Orthop 2015;39:673-80.

20. Aytaç Ş, Atalan G, Gülen G, Yılmaz H. Comparison of femoral nerve block by neurostimulator accompanied with ultrasound and without ultrasound in knee artroplsty. J Clin Anal Med 2015;6:208-11.

21. Moura EC, de Oliveira Honda CA, Bringel RC, Leal Pda C, Filho Gde J, Sakata RK. Minimum Effective Concentration of Bupivacaine in Ultrasound-Guided Femoral Nerve Block after Arthroscopic Knee Meniscectomy: A Randomized, DoubleBlind, Controlled Trial. Pain Physician 2016;19:E79-86.

22. Veneziano G, Tripi J, Tumin D, Hakim M, Martin D, Beltran $\mathrm{R}$, et al. Femoral nerve blockade using various concentrations of local anesthetic for knee arthroscopy in the pediatric population. J Pain Res 2016;9:1073-9.

23. Hartmann FV, Novaes MR, Carvalho MR. Femoral nerve block versus intravenous fentanyl in adult patients with hip fractures a systematic review. Rev Bras Anestesiol 2017;67:67-71.

24. Alzeftawy AE, El-Daba AA. Cold bupivacaine versus magnesium sulfate added to room temperature bupivacaine in sonarguided femoral and sciatic nerve block in arthroscopic anterior cruciate ligament reconstruction surgery. Anesth Essays Res 2016;10:667-73.

25. Liu Q, Chelly JE, Williams JP, Gold MS. Impact of peripheral nerve block with low dose local anesthetics on analgesia and functional outcomes following total knee arthroplasty: a retrospective study. Pain Med 2015;16:998-1006.

26. Park CK, Cho CK, Lee GG, Lee JH. Optimizing dose infusion of $0.125 \%$ bupivacaine for continuous femoral nerve block after total knee replacement. Korean J Anesthesiol 2010;58:468-76.

27. C Wyatt M, Wright T, Locker J, Stout K, Chapple C, Theis JC. Femoral nerve infusion after primary total knee arthroplasty: a prospective, double-blind, randomised and placebo-controlled trial. Bone Joint Res 2015;4:11-6. 